\title{
Phase Diagram of Modulated Structures of Ferroelectric Crystals Based on Quantum Ising Model with Third-Neighbor Interactions
}

\author{
Hiroyuki Mashiyama* \\ Graduate School of Science and Engineering, Yamaguchi University, 1677-1 Yoshida, \\ Yamaguchi 753-8512, Japan
}

(Received May 19, 2015)

\begin{abstract}
Incommensurate-commensurate phase transitions are analyzed using a model derived from the normal coordinate Hamiltonian for a crystal lattice. The Hamiltonian consists of a local self-potential and effective third-neighbor interactions. Free energies of various modulated phases are calculated with a mean-field approximation under the condition that two quantum states within the local potential are important at low temperature. It is demonstrated that the quantum effect works to stabilize the incommensurate phase rather than the commensurate phase. Even at zero temperature, the incommensurate phase can occupy a finite region in the phase diagram. This situation is similar to quantum paraelectricity in some ferroelectrics, and can be expected as a general feature of modulated structures of dielectric crystals. The phase diagram for ferroic first- and third-neighbor interactions but antiferroic second-neighbor interactions is constructed theoretically and is discussed in detail to explain qualitatively the low-temperature behavior of some ferroelectric crystals.
\end{abstract}

\section{Introduction}

Phase transitions of ferroelectric materials are usually described using thermodynamic models based on classical mechanics. The well-known Ising spin model ${ }^{1)}$ or Landau's thermodynamic potential model ${ }^{2)}$ can explain various phase transitions successfully. The order-disorder character of the transition in $\mathrm{KH}_{2} \mathrm{PO}_{4}$ (abbreviated KDP hereafter) was firstly analyzed using the Ising spin model with statistical mechanics. ${ }^{3)}$ To explain the prominent isotope effect in KDP, Blinc proposed the proton tunneling model. ${ }^{4)}$ Soon after, it was pointed out that the tunneling model was equivalent to the Ising spin system in a transverse field. ${ }^{5)}$ Furthermore, the model was represented by using field operators, and the transition of KDP was reinvestigated quantum-mechanically. ${ }^{6}$ ) Thus, the order-disorder transition in KDP was considered to represent the quantum effect afterward. ${ }^{7,8)}$ Another quantum phenomenon in dielectrics is the quantum paraelectricity observed in $\mathrm{SrTiO}_{3}$ and related crystals. ${ }^{9-11)}$ Modified 
thermodynamic potentials have also been proposed to represent thermodynamic quantities in a wide temperature range including low temperature. ${ }^{12)}$ However, the quantum effect in ferroelectrics is limited and usually considered as an exceptional problem.

Incommensurate-commensurate transitions in dielectric crystals attracted much interest a few decades ago. ${ }^{13,14)}$ Phenomenological thermodynamic potential models, ${ }^{15,16)}$ the ANNNI model, ${ }^{17-19)}$ the $\phi^{4}$ model, ${ }^{20)}$ the EXAFII model, ${ }^{21,22)}$ the ELRI model, ${ }^{23)}$ and other models were developed to explain the many types of complex phase diagram consisting of commensurate and incommensurate phases. ${ }^{24,25)}$ It was shown in the DIFFF model ${ }^{26)}$ or in another model that was described by continuous variables that the incommensurate phase did not necessarily transform to the commensurate phase at low temperature (absence of lock-in transition). ${ }^{27)}$

Concerning the stability of commensurate phases, the properties of the two-dimensional classical ANNNI model have been investigated by studying an equivalent quantum spin-chain in a magnetic field. ${ }^{19,28-31)}$ The commensurate structures become rather unstable except for $\mathrm{q}=0,1 / 4$, and $1 / 2$ phases. On the other hand, many commensurate phases with long periods appear, and successive transitions including lock-in transitions proceed in the original ANNNI model in three dimensions. ${ }^{19)}$

Recently, it has been found that the incommensurately modulated structure persists down to very low temperature in ferroelectric crystals. In monoclinic $\mathrm{Rb}_{2} \mathrm{ZnI}_{4}$, the incommensurate structure with $\boldsymbol{q} \cong 0.17 a^{*}-0.30 c^{*}$ appears below $62 \mathrm{~K}^{32)}$ The dielectric susceptibility shows a broad peak at around $5 \mathrm{~K}$, but no ferroelectricity is observed down to $1.5 \mathrm{~K}^{33-35)}$ Such dielectric behavior is similar to the famous quantum paraelectricity in $\mathrm{SrTiO}_{3}$, where ferroelectricity is suppressed by quantum fluctuation. ${ }^{10,11,36)}$ While a ferroelectric transition is induced by the instability of a zone-center optical mode, the stability of a modulated structure is of concern in the general wave number $q$ in incommensurate crystals. To explain the incommensurate phase that survives down to low temperature, we should consider third-neighbor interactions and quantum effect. To the best of the author's knowledge, a quantum three-dimensional system described by Ising variables has not been investigated so far. The phase diagrams are not fully understood, particularly for the model Hamiltonian with more than second-neighbor interactions.

In this work, we study how a phase diagram displaying incommensurate and commensurate phases is affected by quantum effect at low temperature, by employing a model Hamiltonian that describes structural phase transition. ${ }^{37,38)}$ The Hamiltonian is constructed by unit-cell 
coordinates derived from normal coordinates of the harmonic lattice dynamics theory, as explained in Appendix. In Sect. 2, an anharmonic local potential for the local coordinate is added to the Hamiltonian, and effective interactions between cells up to third neighbors along one direction are assumed, in order to discuss the successive transitions from the disordered to incommensurate phases, which may be followed by commensurate phases. At low temperature, we consider that two quantum states in the anharmonic self-potential are essential, and thermodynamic quantities and the free energy are calculated by statistical mechanics using the mean-field theory. It is found that the quantum character becomes real if the energy gap of the two quantum states is comparable to the interaction energy between neighboring cells. ${ }^{36)}$ In our previous paper, only ferroic transition is treated; here, we derive the expressions of the free energy for modulated structures as well.

The free energy for a modulated structure is minimized by numerical iteration methods; the phase diagrams for various interaction parameters are demonstrated in Sect. 3. In the classical limit (the quantum energy gap is zero), the model is reduced to the Ising spin model with third-neighbor interaction, and only five modulated spin alignments with reduced wave numbers $q=0,1 / 2,1 / 3,1 / 4$, and 1/6 are stable at zero temperature. ${ }^{22,39)}$ On the other hand, the order parameter does not increase up to a classical value at low temperature, due to the quantum effect. $^{36)}$ Therefore, an incommensurate state has an advantage in terms of the internal energy at low temperature so that the incommensurate phase may maintain stability down to zero temperature. Strictly speaking, commensurate phases whose periods are longer than 10 are not considered explicitly, and are regarded as if incommensurate in this study, because we are interested in phase transitions of real dielectric crystals and not in high-order commensurate phases with long periods. In the final section, we discuss that calculated results are rather general regardless of the details of the model. We also try to explain the experimentally observed phenomena, with reference to our calculated phase diagrams.

\section{Model and Approximations}

\subsection{Model Hamiltonian}

To discuss one-dimensional modulation, let us consider the model lattice Hamiltonian as described in Appendix:

$$
H=\sum_{j}\left[\frac{1}{2} \dot{x}_{j}^{2}+V\left(x_{j}\right)-h_{j} x_{j}\right]-\frac{1}{2} \sum_{j, l} J_{l} x_{j} x_{j+l}
$$

Here, $x_{j}$ represents a displacement pattern at the $j$-th cell, belonging to a normal mode 
relevant to the structural phase transition. The conjugate external force and self-potential for the variable $x_{j}$ are given by $h_{j}$ and $V\left(x_{j}\right)$, respectively. The anharmonic potential is indispensable for inducing the phase transition. ${ }^{37,38)}$ The self-potential is an even function, since a disordered phase is assumed at high temperature.

In a deep double-well case, the model Hamiltonian is equivalent to the Ising spin system. Such a linear Ising system where $J_{1}>0$ and $J_{2}<0$ is well-known as the ANNNI model displaying various commensurate phases. ${ }^{18,19)}$ On the other hand, the EXAFII model with $J_{1}<0, \quad J_{2}<0$, and third-neighbors interaction $J_{3}$ was investigated to explain the successive transitions in $\mathrm{A}_{2} \mathrm{BX}_{4}$-type crystals. ${ }^{21,22)}$ In this work, we consider that the double well is not so deep that the quantum effect appears. ${ }^{4-6)}$ We shall consider the case of $J_{1}>0$, $J_{2}<0$, and $J_{3}>0$ in detail. Although the displacement variable $x_{j}$ is not a spin variable, the Hamiltonian is equivalent to an Ising model in a transverse field as stated in Sect. 1, ${ }^{5)}$ if a two-level approximation is adopted as described below. Such a quantum system is reduced to the classical Ising model continuously when a quantum parameter is vanishing. Thus, our model may be called a quantum Ising model with third-neighbor interactions (QITNI).

\subsection{Approximations}

Firstly, we define the one-particle Hamiltonian using mean field approximations as

$$
H_{\mathrm{MF}}=\frac{1}{2} \dot{x}_{j}^{2}+V\left(x_{j}\right)-\tilde{h}_{j} x_{j} .
$$

Here, the mean field is written explicitly as

$$
\tilde{h}_{j} \equiv h_{j}+\sum_{l=1}^{3} J_{l}\left(\xi_{j+l}+\xi_{j-l}\right)
$$

and $\xi_{j}=\left\langle x_{j}\right\rangle$ is the expectation value of the order parameter.

If the interactions with neighbors are sufficiently weak (i.e., without the mean field), the quantum state of the "particle" is represented by the wave mechanics equation

$$
\left[-\frac{\hbar^{2}}{2} \frac{\partial^{2}}{\partial x^{2}}+V(x)\right]|m\rangle=\varepsilon_{m}|m\rangle .
$$

The energy $\varepsilon_{m}$ and wave function $|m\rangle$ of the $m$-th state can be calculated analytically or numerically, once the potential is given. If temperature is low, and the ground state $m=0$ and first excited state $m=1$ are separated from $m=2$ or higher states, then the two-state approximation may be sufficient to describe the system. ${ }^{4-6)}$ Under such a condition, the mean 
field is taken into account by perturbation methods, and the energy of the particle in the mean field is given by ${ }^{36)}$

$$
E_{j}^{ \pm}=\frac{\varepsilon_{0}+\varepsilon_{1}}{2} \pm \sqrt{\left(\frac{\varepsilon_{1}-\varepsilon_{0}}{2}\right)^{2}+\left|\left\langle 0\left|\tilde{h}_{j} x\right| 1\right\rangle\right|^{2}} .
$$

The partition function of the $N$-particle system is then written as

$$
Z=\operatorname{Tr} \exp \left(-H_{\mathrm{MF}} / k_{\mathrm{B}} T\right)=\prod_{j}\left[\exp \left(-E_{j}^{-} / k_{\mathrm{B}} T\right)+\exp \left(-E_{j}^{+} / k_{\mathrm{B}} T\right)\right\rfloor,
$$

and the expectation value can be calculated using the mean field Hamiltonian as

$$
\xi_{j}=\frac{\operatorname{Tr} x_{j} e^{-\beta H_{M F}}}{\operatorname{Tr} e^{-\beta H_{M F}}}=k_{B} T \frac{\partial \ln Z}{\partial h_{j}}=\frac{\tilde{h}_{j}}{\Lambda_{j}} x_{0}^{2} \tanh \frac{\Lambda_{j}}{k_{B} T},
$$

where the quantity

$$
\Lambda_{j}=\sqrt{\frac{1}{4}\left(\varepsilon_{1}-\varepsilon_{0}\right)^{2}+\widetilde{h}_{j}^{2} x_{0}^{2}}
$$

contains the mean field; Eq. (7) is the self-consistent equation for determining the order parameter $\xi_{j}$. Here, $x_{0}=\langle 0|x| 1\rangle$ gives a measure of the spread of the wave function of the quantum particle, and is determined by the self-potential width. ${ }^{36)}$

If the external field $h_{j}$ is absent, the disordered state $\left(\xi_{j}=0\right)$ should be a stable phase thermodynamically at high temperature. With decreasing temperature, a spontaneous displacement with the wave number $q$,

$$
\xi_{j}=x_{0} A_{1} \cos 2 \pi q j,
$$

will appear, where the dimensionless amplitude $A_{1}$ is small. Then, Eq. (3) will be approximated as

$$
\tilde{h}_{j} \cong \sum_{l} J_{l} x_{0} A_{1} \cos 2 \pi q(j+l)=x_{0} A_{1} J(q) \cos 2 \pi q j
$$

where $J(q)=\sum_{l=1}^{3} 2 J_{l} \cos 2 \pi q l$ is the Fourier component of the interaction defined by Eq.

(A.7). Hereafter, the energy gap is represented by the temperature $T_{1}$ defined as

$$
T_{1}=\left(\varepsilon_{1}-\varepsilon_{0}\right) / k_{B} \text {. }
$$

Around the transition temperature, the amplitude of the spontaneous displacement should be small; thus, we can write the self-consistent Eq. (7) as

$$
A_{1} \frac{k_{B} T_{1}}{2} \cong A_{1} x_{0}^{2} J(q) \cdot \tanh \frac{T_{1}}{2 T}\left(1+O\left(A_{1}^{2}\right)\right)
$$

Therefore, the spontaneous parameter with the wave number $q$ firstly appears at the 
temperature $T$ that satisfies

$$
\frac{T_{1}}{2 T_{0}(q)}=\tanh \frac{T_{1} / 2}{T},
$$

where the interaction magnitude is represented by the following temperature

$$
T_{0}(q)=J(q) x_{0}^{2} / k_{B} .
$$

Thus, the transition temperature where the order parameter with the wave number $q$ appears spontaneously is given by

$$
T_{\mathrm{c}}(q)=T_{1} / \ln \left\{\left(2 T_{0}(q)+T_{1}\right) /\left(2 T_{0}(q)-T_{1}\right)\right\} .
$$

If the system is classical (i.e., $T_{1}=0$ ), then $T_{\mathrm{c}}(q)=T_{0}(q)$ as expected.

A typical $q$ dependence of the interaction $J(q)$ is shown in Fig. 1. With decreasing temperature, the disordered phase transforms to an ordered phase with the wave number $q_{\max }$, which gives the maximal $J(q)$. As will be described in Fig. 3, a commensurate value of $q_{\max }$ is realized on a straight line in the $J_{2}-J_{3}$ parameter plane; however, the ferroic state $(q=0)$ and the antiferroic state $(q=1 / 2)$ are realized within finite regions in the $J_{2}-J_{3}$ plane.

Here, note that the transition temperature given by Eq. (12) decreases with increasing $T_{1}$. If $T_{1}>2 T_{0}(q)$, then the ordered state becomes unstable down to zero temperature, which is well known as quantum paraelectricity for $q=0 .{ }^{10)}$ Thus, $T_{1}$ is the measure of how the quantum effect works in the system.

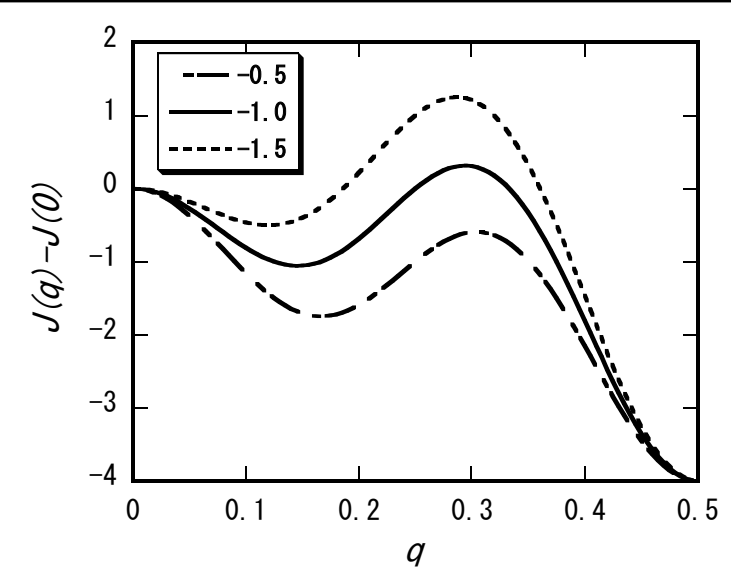

Fig. 1. Fourier component of interaction $J(q)$. Three typical cases with the interaction parameters $J_{1}=0, J_{2}=-0.5$, -1.0 , and -1.5 , and $J_{3}=1$ are drawn, for which $q=0$ (ferro) or $q=q_{\max }$ may be realized at low temperature. 


\subsection{Free energy}

The free energy of the system is calculated from the partition function (6) using mean field approximations as ${ }^{12,36)}$

$$
\begin{aligned}
& F=\frac{1}{2} \sum_{j, l} J_{l} \xi_{j} \xi_{j+l}-k_{B} T \ln Z \\
& =\frac{1}{2} \sum_{j, l} J_{l} \xi_{j} \xi_{j+l}-k_{B} T \sum_{j} \ln \left(2 \cosh \frac{\Lambda_{j}}{k_{B} T}\right)+N \frac{\varepsilon_{0}+\varepsilon_{1}}{2}
\end{aligned} .
$$

Since the energy origin is arbitrary, the last constant term is neglected hereafter.

Simple commensurate structures with $q=0,1 / 2$, and $1 / 4$ are represented by displacement patterns $\uparrow, \uparrow \downarrow$, and $\uparrow \uparrow \downarrow \downarrow$, respectively. Each structure is described by one order parameter, $\eta=\left|\xi_{j}\right| / x_{0} \cdot{ }^{23)}$ The free energy per site of such a structure is represented as

$$
f(q)=\frac{F}{k_{\mathrm{B}} N}=\frac{T_{0}(q)}{2} \eta^{2}-T \ln \left[2 \cosh \left(\frac{\lambda_{q}}{T}\right)\right],
$$

where the mean field is given by

$$
\lambda_{q}=\sqrt{\frac{1}{4} T_{1}^{2}+T_{0}(q)^{2} \eta^{2}} .
$$

With the free energy minimized, the normalized order parameter $\eta$ is given by the following self-consistent equation:

$$
\eta=\frac{T_{0}(q)}{\lambda_{q}} \eta \tanh \left(\frac{\lambda_{q}}{T}\right) .
$$

In the case of $q=0$, the equation was given in a previous paper. ${ }^{36)}$ The same form of Eq. (16) holds for commensurate phases with $q=1 / 4$ and $1 / 2$. In the limit of zero temperature, we obtain the asymptotic values as

$$
\eta \rightarrow \sqrt{1-\left(T_{1} /\left(2 T_{0}(q)\right)\right)^{2}} \text { and } f(q) \rightarrow-\frac{1}{2} T_{0}(q)\left\{1+\left[T_{1} /\left(2 T_{0}(q)\right)\right]^{2}\right\} .
$$

While the order parameter $\eta$ approaches 1 in the classical system of $T_{1}=0$, the value is suppressed in the quantum system of $T_{1}>0$. The ordered phase of the wave number $q$ does not appear down to zero temperature if $T_{1}>2 T_{0}(q)$.

In the following, we adopt a normalized unit such that $k_{\mathrm{B}}$ and $x_{0}$ are unity. The order parameter of the wave number $q$ can be represented by a Fourier series as

$$
\eta_{j}=\sum_{q} \eta_{q} e^{2 \pi i q r_{j}}=A_{1} \cos 2 \pi q j+A_{3} \cos 6 \pi q j+A_{5} \cos 10 \pi q j+\cdots
$$

If $q$ is commensurate, then the series is terminated at the zone center or zone boundary. ${ }^{23)}$ Explicitly, we obtain 


$$
\begin{array}{ll}
q=1 / 3: & \eta_{j}=A_{1} \cos \frac{2 \pi}{3} j+A_{3} \\
q=3 / 10: & \eta_{j}=A_{1} \cos \frac{3 \pi}{5} j+A_{3} \cos \frac{9 \pi}{5} j+A_{5} \cos \pi j \\
q=2 / 7: & \eta_{j}=A_{1} \cos \frac{4 \pi}{7} j+A_{3} \cos \frac{12 \pi}{7} j+A_{5} \cos \frac{20 \pi}{7} j+A_{7} .
\end{array}
$$

The free energy should be minimized with respect to the amplitude $\left\{A_{2 n-1}\right\}$. The concrete equations for $q=2 / 7$ are written as

$$
\begin{aligned}
& \frac{\partial f}{\partial A_{2 n-1}}=T_{0}((2 n-1) q)\left\{\frac{A_{2 n-1}}{2}-\sum_{j=0}^{6} \frac{\cos 2 \pi(2 n-1) q j}{7} \cdot \frac{h_{j}}{\lambda_{j}} \tanh \frac{\lambda_{j}}{T}\right\}=0, \\
& \frac{\partial f}{\partial A_{7}}=T_{0}(0)\left\{A_{7}-\sum_{j=0}^{6} \frac{h_{j}}{7 \lambda_{j}} \tanh \frac{\lambda_{j}}{T}\right\}=0,
\end{aligned}
$$

where

$$
\begin{aligned}
& \lambda_{j}^{2}=\left(\frac{1}{2} T_{1}\right)^{2}+\left(h_{j}\right)^{2} \\
& h_{j}=\sum_{n=1}^{3} T_{0}((2 n-1) q) A_{2 n-1} \cos (2 \pi(2 n-1) q j)+T_{0}(0) A_{7} .
\end{aligned}
$$

Once the amplitudes are calculated, we obtain the free energy $f(q)$ for each $q$ structure. By comparing various $f(q)$ values, we can determine the minimum free-energy phase for the given interaction parameters $\left\{J_{l}\right\}$ and temperature $T$. Actually, the self-consistent equations are solved numerically by iteration methods.

In the case of the classical ANNNI model in three dimensions, many commensurate phases with a long period are recognized in the phase diagram. ${ }^{19,21,22)}$ In principle, we can calculate high-order commensurate phases. ${ }^{23)}$ However, the regions of such commensurate phases are narrow. Thus, we consider an "incommensurate" structure instead of high-order commensurate structures below.

In the case of the incommensurate wave number $q$, we cannot terminate the Fourier series as demonstrated in Eqs. (19) - (21). The harmonics of $q$ are expected to distribute in the range of $(0,2 \pi)$, if the wave number is reduced within $2 \pi$. Therefore, we assume a uniform distribution and replace summation by integration as follows:

$$
f(q)=\frac{1}{4}\left[T_{0}(q) A_{1}^{2}+T_{0}(3 q) A_{3}^{2}+\cdots\right]-\frac{T}{2 \pi} \int_{0}^{2 \pi} d \theta \ln \left[2 \cosh \left(\frac{\lambda}{T}\right)\right],
$$

where 


$$
\begin{aligned}
& \lambda=\sqrt{\left(T_{1} / 2\right)^{2}+h(\theta)^{2}}, \\
& h(\theta)=\sum_{n} T_{0}((2 n-1) q) A_{n} \cos ((2 n-1) \theta) .
\end{aligned}
$$

The extremal condition for Fourier amplitudes is given by

$$
\frac{\partial f}{\partial A_{n}}=T_{0}(n q)\left[\frac{1}{2} A_{n}-\frac{1}{\pi} \int_{0}^{\pi} d \theta \cos (n \theta) \frac{h}{\lambda} \tanh \left(\frac{\lambda}{T}\right)\right]=0 .
$$

The numerical calculation is carried out in the following section.

\section{Phase Diagrams}

\subsection{Low-temperature phases in classical limit}

The Ising spin system with third-neighbor interaction was investigated about 40 years ago. ${ }^{39)}$ Such a system corresponds to the classical limit of our model. The phase diagram at zero temperature is shown in Fig. 2, in agreement with the old paper. ${ }^{22)}$ If the nearest-neighbor interaction is ferroic $\left(J_{1}>0\right)$, there exist six phases with the reduced wave numbers $q=0,1 / 3$, $1 / 4,2 / 5,1 / 6$, and 1/2. Each phase has a region with a finite area. However, the internal energy of the spin alignment with $q=2 / 7$, for example, degenerates to those with $q=1 / 3$ and $1 / 4$ at the boundary between these phases. ${ }^{22)}$

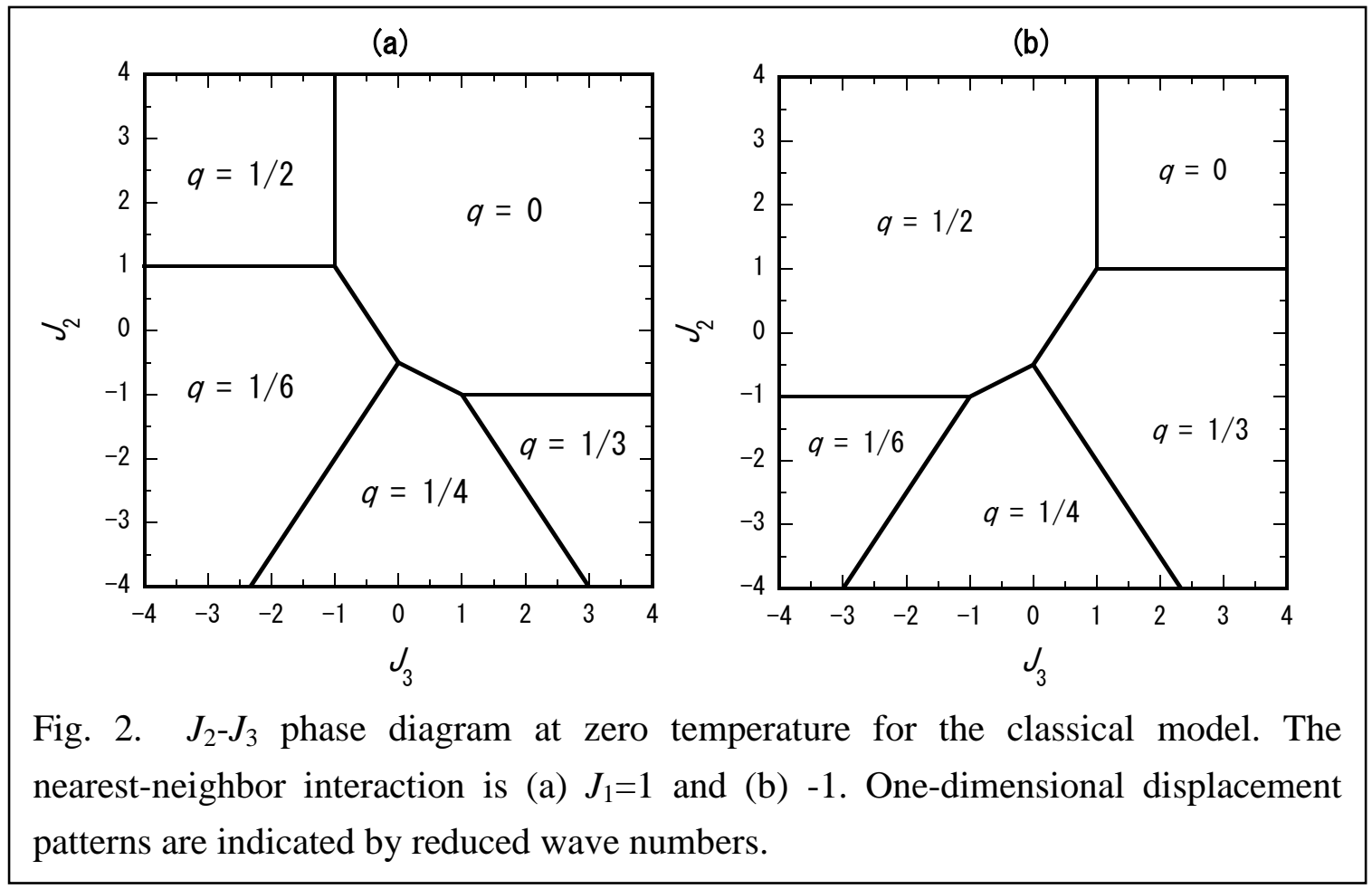

When the nearest-neighbor interaction is antiferroic $\left(J_{1}<0\right)$, the Hamiltonian (the internal energy) is the same as the ferroic one, if the spin alignment is inverted alternatively 
along the modulation and the sign of $J_{3}$ is changed simultaneously. Therefore, the phase diagram for $J_{1}<0$ given in Fig. 2(b) is a mirror reflection of the phase boundaries shown in Fig. 2(a). The wave number $q$ of each phase in Fig. 2(b) is changed by 1/2- $q$ from Fig. 2(a).

The famous ANNNI model considers the cases of $J_{1}>0, J_{2}<0$, and $J_{3}=0 .{ }^{18)}$ On the other hand, the EXAFII model treats the cases of $J_{1}<0, J_{2}>0$, and $J_{3} \neq 0 .{ }^{21,22)}$ In this paper, we are mainly concerned with the case of $J_{1}>0$, since we are interested in modulated phases with $q \sim 0.3{ }^{32)}$ However, our results for $J_{1}>0$ are straightforwardly applied to the system of $J_{1}<0$, by changing the sign of $J_{3}$ and converting $q$ to $1 / 2-q$.

\subsection{Modulation wave number at high temperature}

The ordered structure just below the transition from the disordered phase is represented by a sinusoidal modulation, whose wave number $q$ corresponds to the maximal point of $J(q)$ as described by Eq. (11) at the end of Sect. 2.2. The interaction function $J(q)$ is also extreme at the zone center $q=0$ and the zone boundary $q=1 / 2$. Thus, we have to find the true maximum, and we finally obtain the wave number "contour lines" in the $\left(J_{2}, J_{3}\right)$ parameter space as shown in Fig. 3.

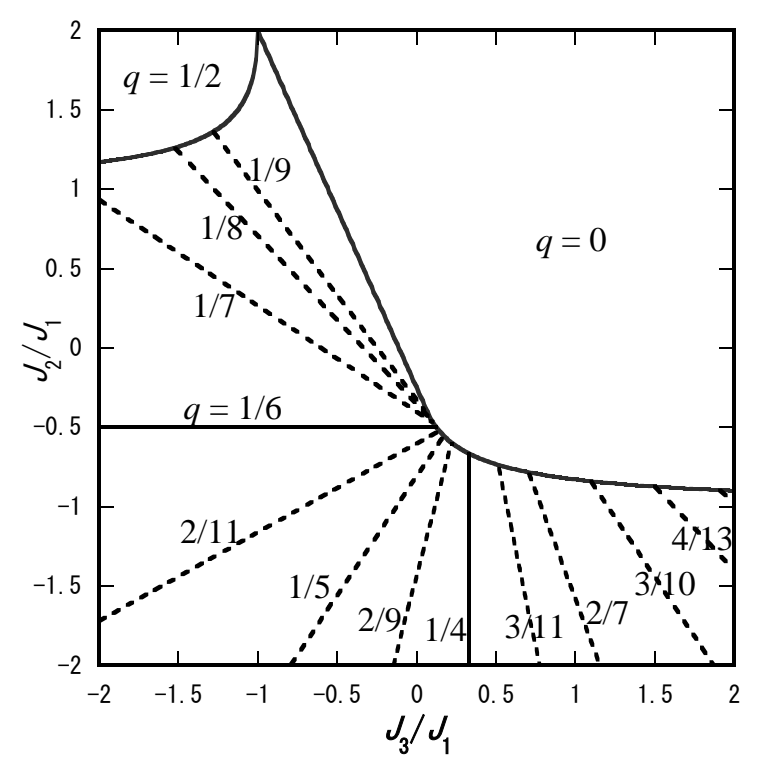

Fig. 3. Contour map of initial wave number at phase transitions from the disordered phase for one-dimensional classical chain model with ferroic nearest-neighbor interaction $\left(J_{1}>0\right)$.

Taking both Figs. 2(a) and 3 into account, we observe that the disordered phase transforms to the ferroic phase $q=0$ if $-1 / 4<J_{2} / J_{1}<0$ or to modulated phases with $q<1 / 3$ if 
$J_{2} / J_{1}<-1 / 4$. Moreover, note that the classical state at $T=0$ is ferroic if $-1 / 2<J_{2} / J_{1}$, and antiferroic with $q=1 / 4$ if $J_{2} / J_{1}<-1 / 2$, and three states with $q=0,1 / 6$, and $1 / 4$ degenerate if $J_{2} / J_{1}=-1 / 2$. These features are known from the analysis of the ANNNI model. ${ }^{18)}$

In the following, we investigate around $J_{2} / J_{1}<-1 / 4$ and $J_{3} / J_{1} \approx 1$ for $J_{1}>0$ in detail, since we are interested in explaining the incommensurate phase of $q<1 / 3$ in $\mathrm{Rb}_{2} \mathrm{ZnI}_{4}$ and related crystals. ${ }^{32)}$

\section{$3.3 T-J_{2}$ phase diagrams}

The free energies of six commensurate phases of $q=0,1 / 2,1 / 3,3 / 10,2 / 7$, and $1 / 4$ are minimized iteratively with respect to the order parameter (the Fourier amplitudes) with the given interaction parameters $J_{2}$ and $J_{3}$. Other commensurate phases should exist; however, each region is generally narrow. Therefore, wave numbers with a large denominator are neglected and we consider such high-order commensurate phases as incommensurate in this work. The wave number $q$ of the incommensurate phase is fixed to the initial value of $q_{\max }$ at the transition from the disordered phase. Five Fourier amplitudes of the modulation are determined numerically by iteration methods. The minimum free energy phase is obtained finally by comparing the free energies of various structures. The accuracy for the incommensurate phase is limited at low temperature, say, $T / J_{1} \leq 0.5$, because of the assumptions to represent the incommensurate structure. Moreover, it is difficult to consider the discommensurated structure in our treatment. Thus, the estimated free energy of the incommensurate phase may be higher than the true value, and the incommensurate phase is disadvantageous somehow to commensurate phases in our numerical result. However, the qualitative feature of the phase diagram might not be changed considerably.

Figures 4-6 show phase diagrams whose interaction parameters are $J_{1}>0,-1.8<J_{2} / J_{1}<-0.6$, and $J_{3} / J_{1}=0.9,1.0$, and 1.1. The phase boundaries for classical $\left(T_{1}=0\right)$ and quantum $\left(T_{1} / J_{1}=1\right)$ are drawn by broken and solid lines, respectively. The phase transitions are first-order ones, except for the transition from the disordered phase. The quantum effect lowers the transition temperature given by Eq. (12).

More remarkable changes become clear at low temperature. The incommensurate region extends to zero temperature with commensurate regions thrust away. In the following, energy is normalized so that $J_{1}=1$. In the classical case of $J_{3}=0.9$ (Fig. 4), two commensurate phases of $q=0$ and $q=1 / 4$ counterchange at $J_{2}=-0.95$ and $T=0$. On the other hand, the incommensurate region occupies the region between these commensurate phases of $q=0$ and $q=1 / 4$, in the 
quantum case of $T_{1}=1$.

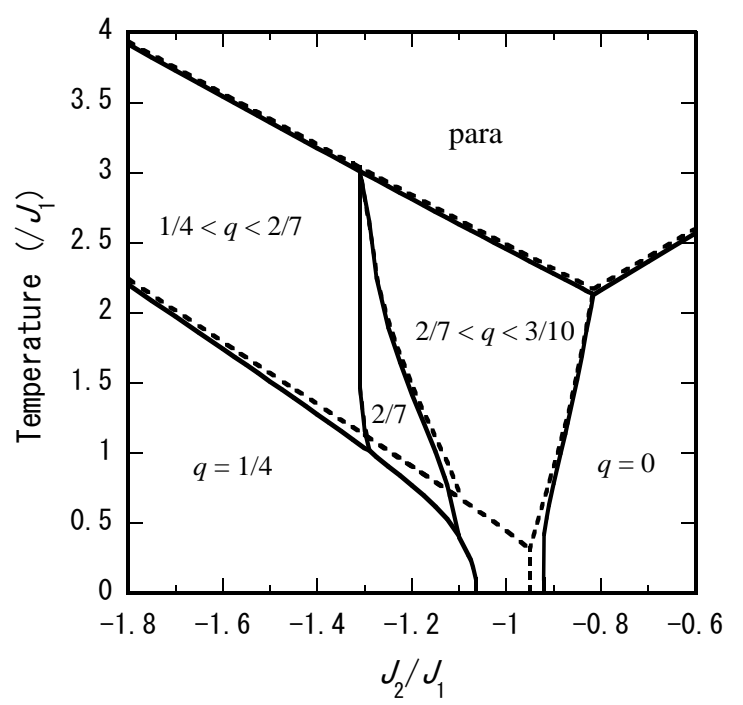

Fig. 4. Phase diagram $T$ vs $J_{2}$ for $J_{1}>0$ and $J_{3} / J_{1}=0.9$.

In the classical case of $J_{3}=1$ (Fig. 5), three phases of $q=0, q=1 / 4$, and $q=$ incommensurate are degenerate at $J_{2}=-1$ and $T=0$. If the quantum effect is introduced, two commensurate phases of $q=0$ and $q=1 / 4$ pull back, while the incommensurate phase extends its region.

In the case of $J_{3}=1.1$ (Fig. 6), another commensurate phase of $q=1 / 3$ appears in the range of $-1.15<J_{2}<-1 \quad\left(T_{1}=0\right)$ between commensurate phases of $q=0$ and $q=1 / 4$. With increasing $T_{1}$, the commensurate phases shrink, and in particular, the $q=1 / 3$ structure disappears perfectly above some critical value of $T_{1}$, which exists between 0.2 and 0.5 .

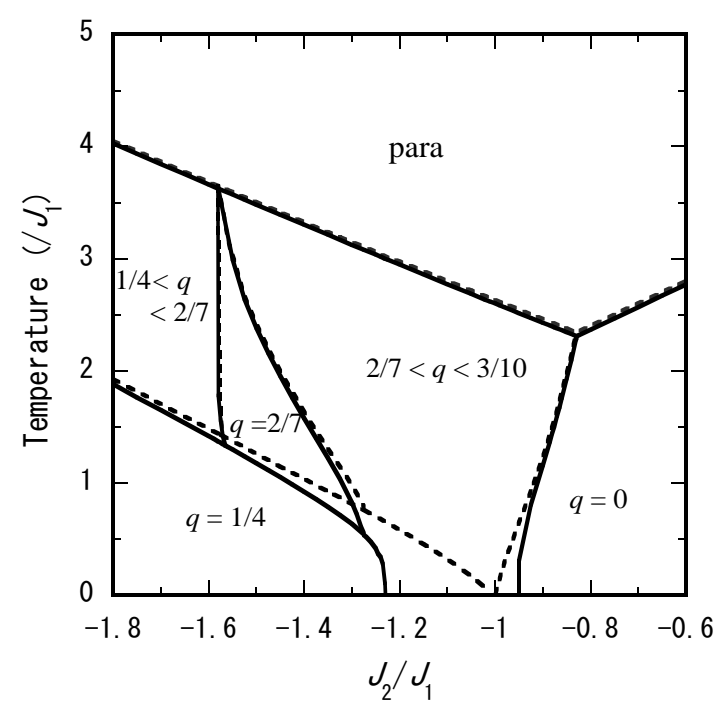

Fig. 5. Phase diagram $T$ vs $J_{2}$ for $J_{1}>0$ and $J_{3} / J_{1}=1.0$. 
The commensurate phase of $q=2 / 7$ is stable in a narrow region, as shown in Figs. 4-6. The shape is similar to that of a bird's bill, as is well known at a temperature below $T_{c}(q)$. The commensurate state of $q=3 / 10$ is metastable even for $J_{3}=1.1$, while the phase appears in a narrow region between the incommensurate and $q=0$ phases for $J_{3}>1.1$.

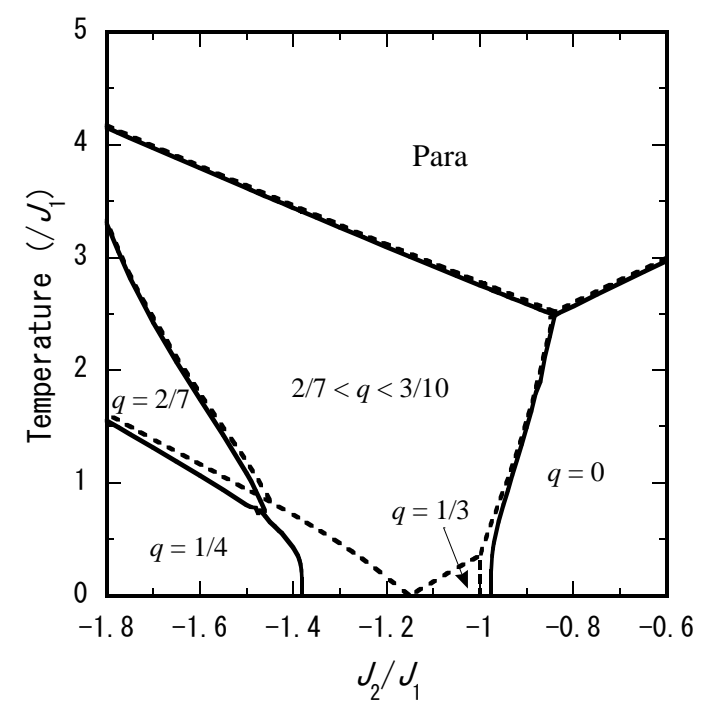

Fig. 6. Phase diagram $T$ vs $J_{2}$ for $J_{1}>0$ and $J_{3} / J_{1}=1.1$.

\section{Conclusions}

Generally, the entropy $S$ of an incommensurate phase is larger than those of commensurate phases, since the degree of freedom is large. Thus, the ordered phase just below the transition from the disordered phase may take an incommensurate wave number. With decreasing temperature, the so-called lock-in energy favors commensurate phases. ${ }^{18-25)}$ At low temperature, the entropy term in the free energy vanishes rapidly, and the internal energy dominates phase stability. In the classical ferroic Ising system with third-neighbor interaction, only low-order commensurate phases with $\mathrm{q}=1 / 6,1 / 4$, and $1 / 3$, as well as ferroic $q=0$ and antiferroic $q=1 / 2$ phases, are stable in finite regions of the phase diagram at $0 \mathrm{~K} .{ }^{22,39)}$

Therefore, it may sound peculiar that the incommensurate phase survives down to $T=0$ in our extended ANNNI model. While the entropies of the commensurate states decrease rapidly at low temperature, the entropy of the incommensurate phase decreases somewhat gradually. However, the entropy contribution to the free energy is small at low temperature. We observe that the internal energy dominates the phase boundary at low temperature. As indicated by Eq. (17), the magnitude of the normalized order parameter $\eta$ is less than unity at $0 \mathrm{~K}$ because of the quantum effect. This situation resembles that of the high-temperature state below $T_{\mathrm{c}}(q)$. 
Therefore, the incommensurate phase can occupy a large area in the phase diagram. The unstable character of a commensurate phase with a long period at $T=0$ was already suggested for the two-dimensional ANNNI model, by soft mode analysis. ${ }^{28-31)}$ The present work shows explicitly the temperature vs $J_{2}$ and $J_{3}$ dependences of phase diagrams for the three-dimensional model which includes the third-neighbor interaction.

In the case of quantum paraelectricity, a ferroelectric transition is blocked at low temperature because of the quantum effect. ${ }^{10)}$ In our model, the transition temperature of Eq. (12) does not exist if the quantum effect is so strong that $T_{1}>2 T_{0}(q)$; neither ferroic, antiferroic, nor other modulated phases exist. On the other hand, if the quantum effect is not so strong that the incommensurate phase and the commensurate phase with $q=1 / 3$ can exist as shown in Fig. 6, the incommensurate phase can survive down to $T=0$.

The incommensurate phase is less stable than the commensurate phase with $q=1 / 3$ in the classical case $\left(T_{1}=0\right)$ at low temperature. However, the former has an advantage over the latter owing to the quantum effect as shown in Fig. 6. We can expect that such a conclusion holds regardless of the details of the model Hamiltonian. In Sect. 3.3, we show the phase diagrams in a narrow region in the $J_{2}-J_{3}$ parameter plane only for $J_{1}>0$. The obtained prediction that the incommensurate state extends its region in the $J_{2}-J_{3}$ parameter plane should be general for a wide range of parameters of $J_{2}$ and $J_{3}$, with the case of $J_{1}<0$ included, if the quantum effect is taken into account.

Here, we note the origin of the rather large third-neighbor interaction $J_{3}$, the same as $J_{1}$ and $J_{2}$. It is not necessarily claimed that the interatomic interaction extends more than the third-neighbor unit cell microscopically. Our interaction parameters $J_{l}$ are defined by Eq. (A.6) and represent not interatomic interactions but effective interactions while reflecting the dispersion relation of the relevant mode. If a modulated structure appears as a result of some complicated interaction between many degrees of freedom, the dispersion relation $\omega^{(s)}(q)$ should take a minimum point at the general wave number $q$, although such a soft mode may not be observed experimentally, because such a mode is often an overdamped one. Then, the wave number-dependent $J(q)$ is maximal, and $J_{2}$ and $J_{3}$ inevitably become as large as $J_{1}$. To represent the dispersion relation $\omega^{(s)}(q)$ accurately, we have to consider a longer range of effective interactions. However, our aim is to explain incommensurate-commensurate transitions at low temperature qualitatively. The wave number dependence, such as that shown in Fig. 1, is essential regardless of the details of the dispersion relation itself. Therefore, we are convinced ourselves with only three effective interactions for simplicity. 
Finally, let us comment on the phase transition of $\mathrm{Rb}_{2} \mathrm{ZnI}_{4}$. The crystal system is monoclinic with the formula unit of $Z=2 .{ }^{40)}$ It transforms from the disordered phase to the incommensurate phase with the modulation wave vector $\boldsymbol{q} \cong 0.17 \boldsymbol{a}^{*}-0.30 \boldsymbol{c}^{*}$ at $62 \mathrm{~K}{ }^{32}$ ) With the actual crystal structure considered, ${ }^{40)}$ this wave vector points almost parallel to the $c$-axis of the unit cell. We can see a pseudo-orthorhombic super cell of $Z=6$ with the common $c$-axis. Thus, we can reveal that the constituent atoms couple ferroic within the $a-b$ plane of the super cell and the interactions along the $c$-axis are competing. Then, our one-dimensional modulation model can be applied to $\mathrm{Rb}_{2} \mathrm{ZnI}_{4}{ }^{41)}$ The magnitude of the modulation wave number is $q_{\mathrm{i}} \cong 0.3$. Thus, the crystal may retain the incommensurate state down to low temperature, if the effective interactions satisfy $J_{2} / J_{1} \cong-1$ and $J_{3} / J_{1} \cong 1$, as shown in Figs. 4-6.

It is reported that the dielectric constant $\varepsilon_{b}$ displayed a remarkable peak at around $5 \mathrm{~K}$; however, no evidence of ferroelectricity was observed down to $1.5 \mathrm{~K}^{33,34)}$ Figures 5 and 6 indicate that the ferroic states of $q=1 / 3$ and $q=0$ may exist in the low-temperature end of the incommensurate state of $q \approx 0.3$, if $T_{1}<1$. Then, the ferroelectric fluctuation may enhance the dielectric constant at low temperature. To investigate this point, the computation of dielectric susceptibility is desired in the near future. In any way, theoretical calculations only demonstrate various possibilities, and a diffraction study at low temperature should be performed to answer the question about the dielectric anomaly and the stability of the incommensurate phase at extremely low temperature.

\section{Acknowledgment}

The author is grateful to Profs. M. Takashige and T. Yamaguchi of Meisei University for many useful discussions about the phase transition of $\mathrm{Rb}_{2} \mathrm{ZnI}_{4}$ and related crystals.

\section{Appendix: Derivation of 1D Hamiltonian from 3D Lattice}

To demonstrate what the displacement variable in Eq. (1) means, we review how to derive the Hamiltonian. According to a standard textbook of solid-state physics, harmonic lattice vibration can be represented by the following Hamiltonian:

$$
H=\frac{1}{2} \sum_{j, k}\left(m_{k} \dot{u}_{k, j}{ }^{2}+\sum_{j, k, j^{\prime}, k^{\prime}} \phi_{k, j, k^{\prime}, j^{\prime}} u_{k, j} u_{k^{\prime}, j^{\prime}}\right) \text {. }
$$

Here, $u_{k, j}$ is a small deviation of the $k$-th atom with the mass $m_{k}$ in the $j$-th unit cell from its 
equilibrium position. Actually, the variable is a vector component; we neglect vector notations to avoid complexity in this report. In Eq. (A.1), bilinear interactions are represented by $\phi_{k, j, k^{\prime}, j^{\prime}}$. The Hamiltonian can be diagonalized by the famous linear transformation:

$$
u_{k, j}=\frac{1}{\sqrt{N m_{k}}} \sum_{q, s} Q_{q}^{(s)} e_{k, s}(q) \mathrm{e}^{2 \pi i q R_{j}},
$$

where $e_{k, s}(q)$ is an eigenvector of the so-called dynamical matrix for the wave number $q$ and belongs to an irreducible representation specified by the label $s$. The familiar form of the harmonic Hamiltonian is rewritten using the eigenvalues $\omega^{(s)}(q)$ of lattice vibration as

$$
H=\frac{1}{2} \sum_{q, s}\left(\dot{Q}^{(s)} \dot{Q}^{(s)}{ }_{-q}+\omega^{(s)}(q)^{2} Q_{q}^{(s)} Q^{(s)}{ }_{-q}\right) .
$$

Usually, one mode is relevant to a phase transition; ${ }^{2)}$ thus, we consider only this normal mode and define the displacement $x_{j}$ at the $j$-th cell as the representative of the relevant mode of the phase transition;

$$
x_{j}=\frac{1}{\sqrt{N}} \sum_{q} Q_{q}^{(s)} \mathrm{e}^{2 \pi i q R_{j}} .
$$

With the local variable of (A.4), Eq. (A.3) is rewritten as

$$
H=\frac{1}{2} \sum_{j}\left(\dot{x}_{j}^{2}-\sum_{l} J_{l} x_{j} x_{j+l}\right),
$$

where the effective interactions between cells are defined as

$$
J_{l}=-\frac{1}{N} \sum_{q} \omega^{(s)}(q)^{2} \exp \left(-2 \pi i q\left(R_{j}-R_{j+l}\right)\right) .
$$

The inversion symmetry $J_{-l}=J_{l}$ is assumed, and we consider that the modulated structures can be represented by the one-dimensional wave number $q$. Then, the Fourier transformation of the effective interaction is given explicitly by

$$
J(q)=2 \sum_{l=1} J_{l} \cos 2 \pi q l
$$

where the wave number $q$ is a reduced one.

However, a phase transition does not take place in such a quadratic Hamiltonian as Eq. (A.5). Some anharmonicity is necessary. We follow Lines and Glass ${ }^{38)}$ and add to Eq. (A.5) an anharmonic self-potential $V\left(x_{j}\right)$, which is an even function to ensure a disordered phase at high temperature. Thus, we derive the model Hamiltonian Eq. (1), which is widely introduced in prominent monographs. ${ }^{37,38)}$ We suppose that the crystal consists of a stacked layer with 
ferroic intralayer interaction, and effective interlayer interactions $J_{l}$ are defined using Eq. (A.6). If the interlayer interactions are competing ones, then one-dimensional modulated structures may appear.

*E-mail: mashi@yamaguchi-u.ac.jp

1) E. Ising, Z. Phys. 31, 253 (1925).

2) L. D. Landau and E. M. Lifshitz, Statistical Physics, translated by J. B. Sykes and M. J. Kearsley (Pergamon Press, New York, 1980) 3rd ed.,Chap.13.

3) J. C. Slater, J. Chem. Phys. 9, 16 (1941).

4) R. Blinc, J. Phys. Chem. Solids 13, 204 (1960).

5) P. G. de Gennes, Solid State Commun. 1, 132 (1963).

6) M. Tokunaga and T. Matsubara, Prog. Theor. Phys. 35, 581 (1966).

7) K. K. Kobayashi, J. Phys. Soc. Jpn. 24, 497 (1968).

8) S. E. Mkam Tchouobiap and H. Mashiyama, Phys. Rev. B 76, 014101 (2007).

9) J. H. Barrett, Phys. Rev. 86, 118 (1952).

10) K. A. Muller and H. Burkard, Phys. Rev. B 19, 3593 (1979).

11) S. E. Mkam Tchouobiap and H. Mashiyama, J. Phys.: Condens. Matter 20, 055223 (2008).

12) E. K. H. Salje, B. Wruck, and H. Thomas, Z. Phys. B: Condens. Matter 82, 399 (1991).

13) J. D. Axe, M. Iizumi, and G. Shirane, in Incommensurate Phases in Dielectrics, ed. R. Blinc and A. P. Levanyuk (North-Holland, Amsterdam, 1986) Vol. 2, Chap. 10, p. 1.

14) H. Z. Cummins, Phys. Rep. 185, 211 (1990).

15) A. P. Levanyuk, in Incommensurate Phases in Dielectrics, ed. R. Blinc and A. P. Levanyuk (North-Holland, Amsterdam, 1986) Vol. 1, Chap. 1, p. 1.

16) Y. Ishibashi, in Incommensurate Phases in Dielectrics, ed. R. Blinc and A. P. Levanyuk (North-Holland, Amsterdam, 1986) Vol. 2, Chap. 11, p. 49.

17) R. J. Elliott, Phys. Rev. 124, 346 (1961).

18) P. Bak and J. von Boehm, Phys. Rev. B21, 5297 (1980).

19) W. Selke, Phys. Rep. 170, 213 (1988).

20) T. Janssen and J. A. Tjon, Phys. Rev. B 24, 2245 (1981).

21) Y. Yamada and N. Hamaya, J. Phys. Soc. Jpn. 52, 3466 (1983).

22) W. Selke, M. Barreto, and J. Yeomans, J. Phys. C: Solid State Phys. 18, L393 (1985).

23) H. Mashiyama, J. Phys. C: Solid State Phys. 16, 187 (1983).

24) H. Mashiyama, J. Phys. Soc. Jpn. 49, 2270 (1980).

25)Z. Y. Chen and M. B. Walker, Phys. Rev. B 43, 5634 (1991).

26) T. Janssen and A. Janner, Adv. Phys. 36, 519 (1987).

27) C. Benkert, V. Heine, and E. H. Simmons, Europhys. Lett. 3, 833 (1987).

28) P. Sen and B. K. Chakrabarti, Phys. Rev. B 40, 760 (1989). 
29) C. M. Arizmendi, A. H. Rizzo, L. N. Epele, and C. A. Garcia Canal, Z. Phys. B Condensed Matter 83, 273 (1991).

30) P. Sen, S. Chakraborty, S. Dasgupta, and B. K. Chakrabarti, Z. Phys. B - Condensed Matter 88, 333 (1992).

31) A. Dutta and D. Sen, Phys. Rev. B 67, 094435 (2003).

32) J. Puragahn, Ph. D. Thesis, Karlsruhe Univ., ISBN 3-932136-37-3 (1998).

33) M. Takashige, T. Yamaguchi, and F. Shimizu, J. Phys. Soc. Jpn. 81, 045001 (2012).

34) T. Yamaguchi, F. Shimizu, and M. Takashige, Ferroelectrics 462, 104 (2014).

35) R. Shibata, R. Muraki, T. Yamaguchi, and M. Takashige, J. Korean Phys. Soc. 66, 1367 (2015).

36) H. Mashiyama, S. E. Mkam Tchouobiap, and M. Ashida, J. Phys. Soc. Jpn. 77, 084709 (2008).

37) R. Blinc and B. Zeks, Soft Modes in Ferroelectrics and Antiferroelectrics (North-Holland, Amsterdam, 1974) Chap. 5.

38) M. E. Lines and A. M. Glass, Principles and Applications of Ferroelectrics and Related Materials (Oxford University Press, Oxford, U.K., 1977) Chap. 2.

39) S. Katsura and A. Narita, Prog. Theor. Phys. 50, 1750 (1973).

40) H. Kasano, Ph. D. Thesis, School of Sci. and Eng., Yamaguchi University, Yamaguchi (2003).

41) H. Mashiyama, presented at the 13th Int. Meeting on Ferroelectricity, Krakow, Poland, 2013. 